\title{
Estimation of critical and viscous frequencies for Biot theory in cancellous bone
}

\author{
Elinor R. Hughes ${ }^{a}$, Timothy G. Leighton ${ }^{\mathrm{a}, *}$, Graham W. Petley ${ }^{\mathrm{b}}$, \\ Paul R. White ${ }^{\text {a }}$, Robert C. Chivers ${ }^{\text {a }}$ \\ ${ }^{a}$ Institute of Sound and Vibration Research, University of Southampton, Southampton SO17 1BJ, UK \\ ${ }^{\mathrm{b}}$ Department of Medical Physics and Bioengineering, Southampton University Hospitals NHS Trust, Tremona Road, Southampton SO16 6 YD, UK
}

Received 15 August 2001; accepted 14 March 2003

\begin{abstract}
The use of Biot theory for modelling ultrasonic wave propagation in porous media involves the definition of a 'critical frequency' above which both fast and slow compressional waves will, in principle, propagate. Critical frequencies have been evaluated for healthy and osteoporotic cancellous bone filled with water or marrow, using data from the literature. The range of pore sizes in bone gives rise to a critical frequency band rather than a single critical frequency, the mean of which is lower for osteoporotic bone than normal bone. However, the critical frequency is a theoretical concept and previous researchers considered a more realistic 'viscous frequency' above which both fast and slow waves may be experimentally observed. Viscous frequencies in bone are found to be several orders of magnitude greater than calculated critical frequencies. Whereas two waves may well be observed at all ultrasonic frequencies for water-filled cancellous bone at $20{ }^{\circ} \mathrm{C}$, it is probable megahertz frequencies would be needed for observation of two waves in vivo.
\end{abstract}

(C) 2003 Elsevier Science B.V. All rights reserved.

PACS: 43.80.Cs; 43.20.Jr; 43.35.Cg

Keywords: Ultrasonic bone assessment; Biot's theory; Cancellous bone; Trabecular bone; Critical frequency

\section{Introduction}

Ultrasonic techniques for the non-invasive detection of osteoporosis have received considerable attention during the last decade. There has been an increasing tendency to move from empirically based approaches to those that have a sound theoretical foundation [1]. Cancellous bone is a complex material that demands sophisticated approaches if the basic phenomenology is to be modelled adequately. Among the models that have been discussed in this context is that of Biot's theory for porous media [2]. A detailed summary of its application to bone has been given by Hughes et al. [1]. The theory distinguishes between propagation in low and high frequency ranges, which are divided by a "critical frequency'. However, a second parameter called the

\footnotetext{
${ }^{*}$ Corresponding author. Tel.: +44-23-8059-2291; fax: +44-23-80593190.

E-mail address: tgl@soton.ac.uk (T.G. Leighton).
}

'viscous frequency' may be of greater practical value. The present note discusses the evaluation of these frequencies for normal and osteoporotic cancellous bone.

\section{Viscous skin depth and the critical frequency}

An essential element of Biot's theory [2] is the prediction of the propagation of two types of compressional wave in porous media. In the first (fast) wave the fluid and (porous) matrix are locked together and move in phase. The locking can arise through the action of one of two dynamic coupling forces: viscous or inertial. The second kind of wave (the 'slow' wave) requires relative motion between the fluid in the pores and the matrix. Biot's theory assumes that the motion of the pore fluid, relative to the solid, follows Poiseuille flow.

A viscous fluid in Poiseuille flow in a cylindrical tube is characterised by a parabolic velocity gradient in a direction perpendicular to the tube wall [3]. For stationary 
pore walls, in general at a distance $y$ from the wall, the fluid velocity parallel to the wall, $\dot{u}$, is

$\dot{u}=\dot{u}_{\max } \exp \left[j \omega t-(1+j)\left(\frac{\omega \rho}{2 \eta}\right)^{1 / 2} y\right]$

for maximum velocity, $\dot{u}_{\max }$; fluid shear viscosity, $\eta$; fluid density, $\rho$; and angular frequency, $\omega$. If the wall oscillates harmonically in a direction parallel to the length of the tube, a disturbance is set up in the fluid. This has the form of an harmonic wave travelling in the $y$ direction whose amplitude decays exponentially with distance from the wall. For different profiles of viscous flow, boundary layers are often based on the distance where the velocity attains a certain percentage of its maximum value [4]. For Poiseuille flow in a porous medium the characterising boundary layer is known as the viscous skin depth, $d$, and is expressed as

$d=(2 \eta / \omega \rho)^{1 / 2}$

The viscous skin depth is frequency dependent. Poiseuille flow only occurs when the viscous skin depth is greater than the pore radius, $r$. The assumption of a parabolic flow profile fails when the skin depth is equal to or less than the pore radius. The equality therefore represents a key condition that occurs at a particular frequency known as the 'critical frequency', $\omega_{\text {crit }}$,

$\omega_{\text {crit }}=2 \eta / \rho r^{2}$

Fig. 1 summarises the frequency regimes for Biot's theory, which is applicable at wavelengths much longer than the pore size. In the low frequency range $\left(\omega<\omega_{\text {crit }}\right.$ for $d>r$ ) Poiseuille flow occurs. The locking of fluid and solid motion arises from the fluid viscosity, resulting

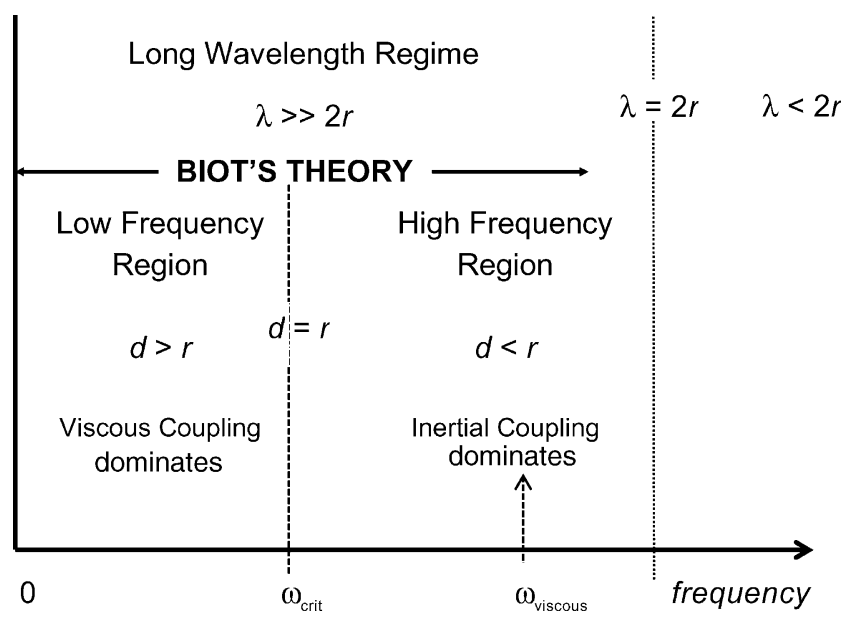

Fig. 1. Frequency regimes in Biot's theory, showing regions differentiated by the value of ratios between viscous skin depth, $d$, wavelength, $\lambda$; pore radius, $r$. in propagation of a fast wave. The relative motion of solid and fluid required for slow wave propagation cannot be realised in this frequency range. The oscillation of the matrix yields a disturbance in the fluid characterised by a diffusion equation [5].

Above the critical frequency $\left(\omega>\omega_{\text {crit }}, \lambda \gg r, d<r\right)$ Poiseuille flow is not established. The relative motion of the solid and fluid is not impeded by viscous drag so that the slow wave can propagate. In this regime viscous coupling is dominated by inertial coupling which locks the solid and the fluid together, as required for fast wave propagation. Inertial coupling occurs when a solid body accelerates in a fluid and drags with it an additional body of surrounding fluid. The amount of fluid involved in this process depends on the porosity and surface area of the matrix. Biot discussed the effect in terms of a parameter called the tortuosity. The latter is a key factor and various expressions have been derived for its evaluation [6-9]. Many relate to matrix geometry, whilst other factors introduce frequency dependent effects.

Thus above the critical frequency both fast and slow waves will propagate and may, in principle, be observed. In practice however, Johnson and Plona found that in some porous media, the slow wave could only be observed if the viscous skin depth was considerably less than the pore size [5]. They devised a second limit, known as the 'viscous frequency', $\omega_{\text {viscous }}$,

$\omega_{\text {viscous }}=\frac{2 \eta}{\rho r^{2} \zeta^{2}}$

where $\zeta$ is the scaling constant with an empirically derived value of the order of 0.01 .

\section{Evaluation of the critical frequency and phase velocities for cancellous bone}

Eqs. (3) and (4) show that changes in both matrix geometry and fluid viscosity, $\eta$, will affect critical and viscous frequencies, $\omega_{\text {crit }}$ and $\omega_{\text {viscous. }}$. The analysis above uses a single value for the pore radius. An individual skeletal site will contain a range of pore sizes, and such a range will result in a critical band rather than a single value of $\omega_{\text {crit }}$. Furthermore, there is a variation in the average pore sizes between individuals in the population. Mellish et al. [10] gives mean values and standard deviations within groups of 15 females, with typical pore radii of $285 \pm 50 \mu \mathrm{m}$ for young normal bone, and $455 \pm 130 \mu \mathrm{m}$ for aged or osteoporotic bone. Such variations will result in a range of $\omega_{\text {crit }}$ between, and within, population groups. Another effect is that the presence of interstitial marrow rather than water (the usual in vitro case) significantly affects ultrasonic propagation in bone, owing to its higher viscosity $[11,12]$.

Fig. 2 shows the viscous skin depth as a function of frequency for water at $20^{\circ} \mathrm{C}(\eta=0.001 \mathrm{Pas}$ [13]), and 


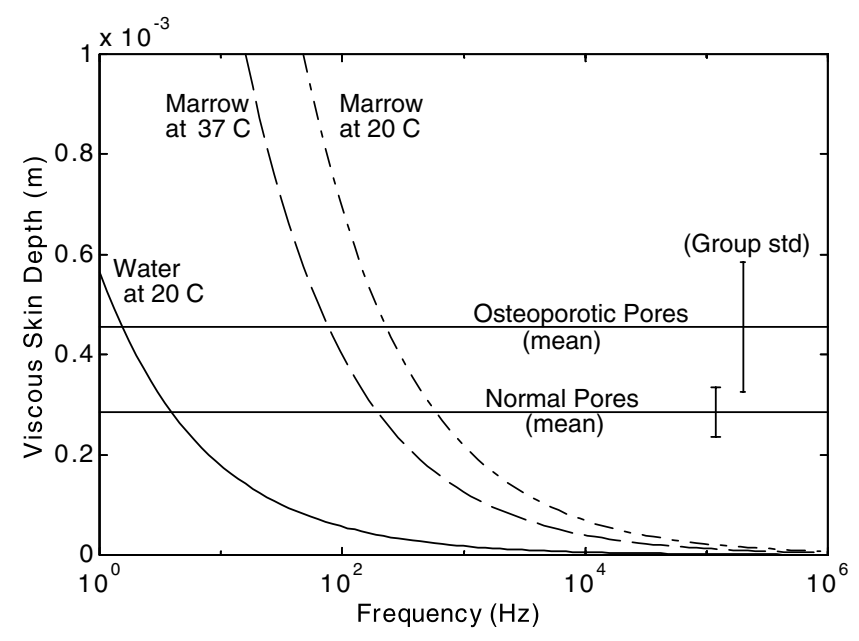

Fig. 2. Viscous skin depth versus frequency, for marrow at 20 and 37 ${ }^{\circ} \mathrm{C}$ and water at $20{ }^{\circ} \mathrm{C}$, with means and standard deviations of pore radii for normal and osteoporotic bone.

Table 1

Critical frequencies for normal and osteoporotic group, in bone with different fluids

\begin{tabular}{lll}
\hline & \multicolumn{2}{l}{ Critical frequency range (mean) } \\
\cline { 2 - 3 } & Normal group & Osteoporotic group \\
\hline Water at $20{ }^{\circ} \mathrm{C}$ & $3.3-6.9 \mathrm{~Hz}(4.5 \mathrm{~Hz})$ & $0.1-2.9 \mathrm{~Hz}(1.4 \mathrm{~Hz})$ \\
Marrow at $37^{\circ} \mathrm{C}$ & $156-341 \mathrm{~Hz}(224 \mathrm{~Hz})$ & $48-148 \mathrm{~Hz}(76 \mathrm{~Hz})$ \\
Marrow at $20^{\circ} \mathrm{C}$ & $490-1042 \mathrm{~Hz}(705 \mathrm{~Hz})$ & $143-451 \mathrm{~Hz}(231 \mathrm{~Hz})$ \\
\hline
\end{tabular}

for marrow at 20 and $37{ }^{\circ} \mathrm{C}$. Since viscosity values of human marrow were not available, values for bovine marrow at $20{ }^{\circ} \mathrm{C}(\eta=0.15 \mathrm{Pas})$ and $37{ }^{\circ} \mathrm{C}(\eta=0.05$ $\mathrm{Pas}$ ) were used [14]. In all cases a fluid density, $\rho$, of $1000 \mathrm{~kg} \mathrm{~m}^{-3}$ was assumed. Also shown are the means and standard deviations (within population groups) of pore radii for normal and osteoporotic bone. The ranges of $\omega_{\text {crit }}$, calculated from Eq. (3) are given in Table 1, whilst corresponding values of $\omega_{\text {viscous }}$ are the table values multiplied by a factor of $10^{4}$. Fig. 3 shows fast and slow wave phase velocities evaluated from Biot's theory using the method in Ref. [9], for normal bone filled with water or marrow at $20{ }^{\circ} \mathrm{C}$. Each wave is represented by two curves: at $1 \mathrm{MHz}$, the upper curve in each case is for osteoporotic bone, whilst the lower curve is for normal bone [9].

\section{Discussion}

Clearly, changes in pore size and the interstitial fluid viscosity both affect the critical and viscous frequencies in bone. First, the greater the viscosity, the higher are these frequencies. For normal bone, in theory, both fast and slow waves will propagate in cancellous bone at
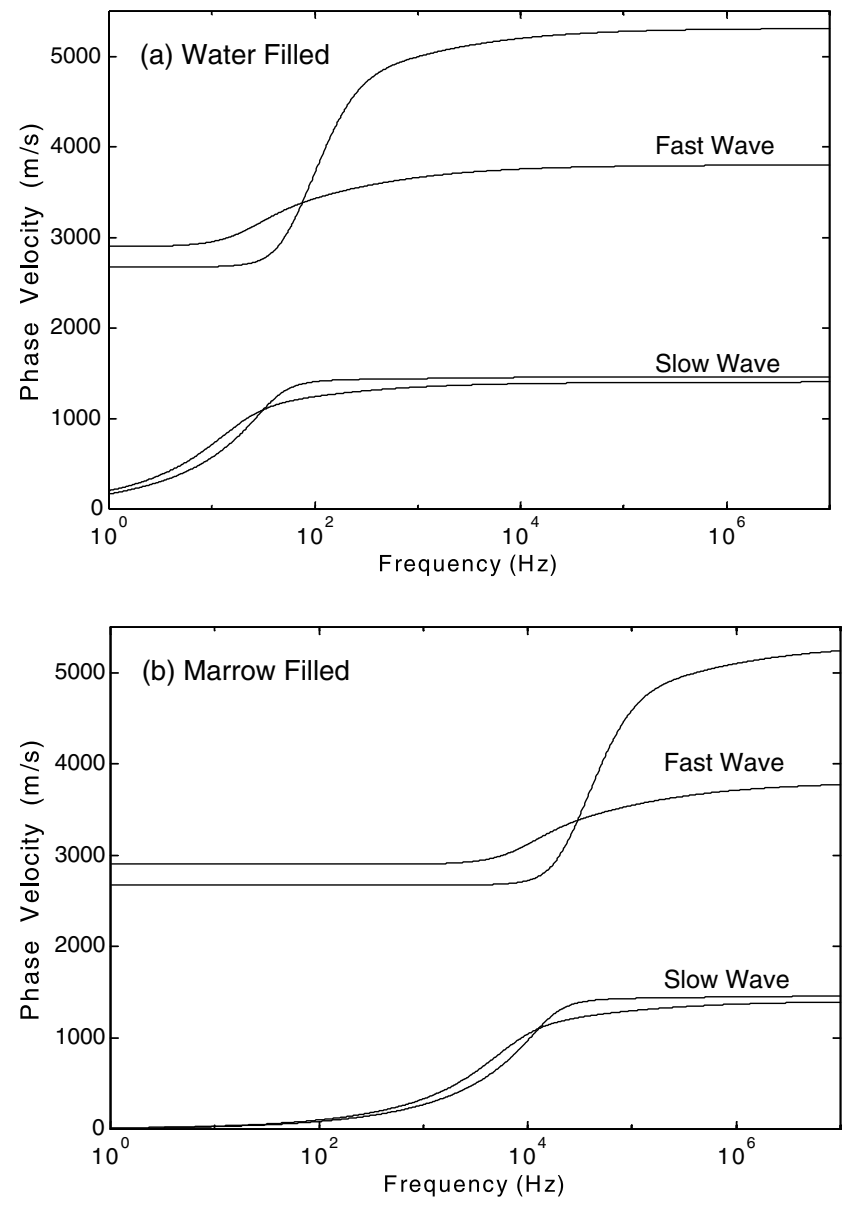

Fig. 3. Phase velocities of fast and slow waves predicted by Biot's theory for cancellous bone at $20^{\circ} \mathrm{C}$ : (a) water filled (b) marrow filled. Each wave is represented by two curves: at $1 \mathrm{MHz}$, the upper curve in each case is for osteoporotic bone, whilst the lower curve is for normal bone.

frequencies above about $\omega_{\text {crit }}=700 \mathrm{~Hz}$. However in practice, their observation may require working frequencies significantly greater than this. For the usual in vitro condition (water-filled bone at $20^{\circ} \mathrm{C}$ ) both waves should be seen above about $\omega_{\text {viscous }}=40 \mathrm{kHz}$ (i.e. most ultrasonic frequencies). In contrast, the in vivo case (marrow-filled bone at $37{ }^{\circ} \mathrm{C}$ ) yields $\omega_{\text {viscous }}=2 \mathrm{MHz}$. This emphasises the importance of the presence of marrow in vivo when attempting the clinical application of in vitro results. Since the in vivo limit is above the bandwidth of clinical testing $(200-600 \mathrm{kHz})$, it could explain why fast and slow waves have not been confirmed in vivo. However, the fact that both waves were seen in vitro at frequencies below $\omega_{\text {viscous }}=7 \mathrm{MHz}$ in marrow-filled bone [1], is a reminder that Eq. (4) is an empirical rule.

Second, larger-pored osteoporotic bone yields lower values of $\omega_{\text {crit }}$ and $\omega_{\text {viscous }}$ than normal bone for the same fluid conditions. However, the curvature of the graphs in Fig. 2 (on a logarithmic scale) causes the range 
of $\omega_{\text {crit }}$ within a population group, to be narrower for osteoporotic bone, even though pore distributions are wider than for normal bone. In the case of a spread of pore size at a specific skeletal site, where there exist bands of $\omega_{\text {crit }}$ and $\omega_{\text {viscous }}$, the only statement that can be made with any certainty is that onset of slow wave propagation will be expected to occur above the upper limit of a band, determined by the small-pore sizes in the distribution.

The variation of critical and viscous frequencies with pore size suggests the idea of an instrument with carefully controlled detection levels and specific frequency. With this, the detection of a slow wave could, per se, be used as a positive indication of a certain level of osteoporosis (measured in terms of mean pore size).

\section{Acknowledgement}

Elinor Hughes was financially supported by EPSRC (UK), Grant \#GR/M37806.

\section{References}

[1] E.R. Hughes, T.G. Leighton, G.W. Petley, P.R. White, Ultrasound Med. Biol. 25 (5) (1999) 811-821.

[2] M.A. Biot, J. Acoust. Soc. Am. 28 (2) (1956) 168-178, 179-191.

[3] J.W. Daily, D.R.F. Harleman, Fluid Dynamics, Addison-Wesley, Massachusetts, 1966.

[4] F.S. Sherman, Viscous Flow, McGraw-Hill, London, 1990

[5] D.L. Johnson, T.J. Plona, J. Acoust. Soc. Am. 72 (2) (1982) 556565.

[6] K. Attenborough, J. Acoust. Soc. Am. 81 (1) (1987) 93-103.

[7] D.L. Johnson, J. Koplik, R. Dashen, Fluid Dyn. 176 (1987) 379 402.

[8] J.G. Berryman, Appl. Phys. Lett. 37 (1980) 382-384.

[9] E.R. Hubbuck, PhD Thesis, Southampton University, 2000.

[10] R.W.E. Mellish, N.J. Garrahan, J.E. Compston, Bone Miner. 6 (1989) 331-338.

[11] J.M. Alves, J.T. Ryaby, J.J. Kaufman, F.P. Magee, R.S. Siffert, Calcif Tissue Int. 58 (1996) 362-367.

[12] P.H.F. Nicholson, Private communication, 2000.

[13] L.E. Kinsler, A.R. Frey, A.B. Coppens, J.V. Sanders, Fundamentals of Acoustics, third ed., John Wiley and Sons, Canada, 1982.

[14] J.D. Bryant, T. David, P.H. Gaskell, S. King, G. Lond, Proc. Inst. Mech. Eng. 203 (1989) 771-775. 